\title{
Tuning Chirality in Photonic Metamaterials with Semiconductor Quantum Dots
}

\author{
E. Plum ${ }^{1, \# \text {, X.-X. Liu }}{ }^{1,2}$, V. A. Fedotov ${ }^{1}$, Y. Chen ${ }^{3}$, D. P. Tsai ${ }^{2}$ and N. I. Zheludev ${ }^{1}$ \\ ${ }^{1}$ Optoelectronics Research Centre, University of Southampton, SO17 1BJ, UK \\ ${ }^{2}$ Department of Physics, National Taiwan University, Taipei, 106, Taiwan, Republic of China \\ ${ }^{3}$ Central Microstructure Facility, Rutherford Appleton Laboratory, Oxfordshire, OX11 OQX, UK \\ ${ }^{\#}$ Email: erp@orc.soton.ac.uk,web: http://www.nanophotonics.org.uk/nizJ
}

Recently we have shown that the classical phenomenon of optical activity, which is traditionally associated with chirality (helicity) of organic molecules, proteins and inorganic structures, can be observed in artificial planar media which exhibit neither 3D nor 2D chirality and called this effect "extrinsic chirality", see Fig. 1 (a) and (b). Here chirality is derived from the mutual orientation of the light beam and the metamaterial array. We observed the effect in the microwave part of the spectrum at oblique incidence to regular arrays of non-chiral subwavelength meta-molecules in form of strong circular dichroism and birefringence indistinguishable from those of chiral three-dimensional media [1].

Here for the first time using a photonic metamaterial consisting of a regular array of nanoscale asymmetrically split ring resonators we demonstrate strong "extrinsic chirality" in the optical part of the spectrum. Moreover we show that the resonant optical activity can be efficiently tuned by hybridizing the metamaterial with semiconductor quantum dots.

The metamaterial structure consists of aluminium nano-wires on glass and was manufactured by electron beam lithography. At oblique incidence, when the experimental arrangement becomes extrinsically chiral, we observed that the nanostructure becomes more transparent for one circular polarization than the other. This transmission difference or circular dichroism is reversed for enantiomeric arrangements, i.e. opposite angles of incidence $\pm \alpha$, see Fig. 1 (c). Importantly, we found that the spectral localization of the chiral material response can be tuned substantially by hybridization with a thin layer of $\mathrm{PbS}$ quantum dots.

Quantum dots deposited on the metamaterial can be optically pumped, which may lead to an enhancement of the effect and reduced losses. These possibilities will be discussed at the conference.

(a)

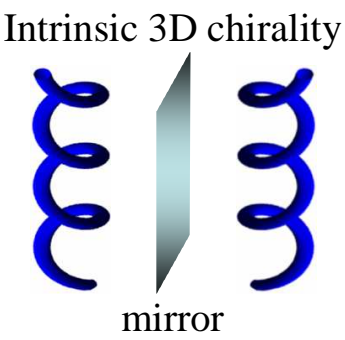

(b) Extrinsic 3D chirality

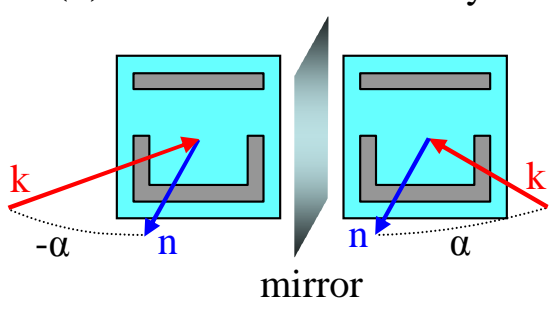

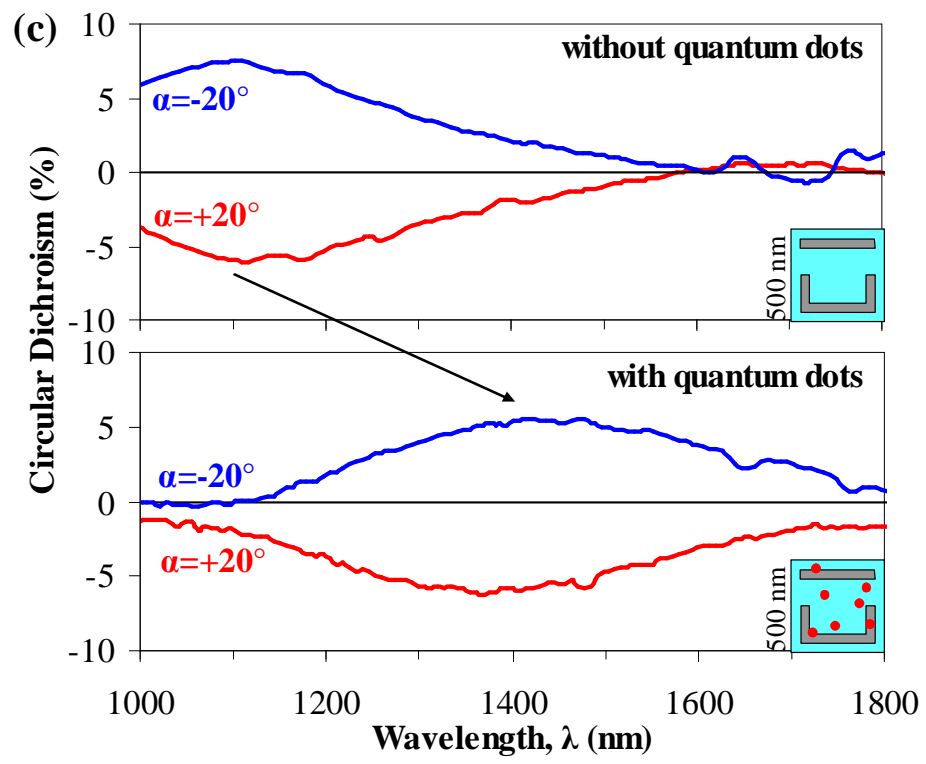

Fig. 1 (a) Intrinsically chiral structure. (b) Achiral metamaterial unit cell, which can become extrinsically chiral at oblique incidence. Two enantiomeric configurations are shown. The structure consists of aluminum nano-wires on glass. (c) Spectral dispersion of circular dichroism measured for the photonic metamaterial at different angles of incidence $\alpha$. Deposition of a thin layer of quantum dots shifts the spectral response substantially.

\section{References}

[1] E. Plum, V. A. Fedotov, and N. I. Zheludev, "Optical activity in extrinsically chiral metamaterial,” Appl. Phys. Lett. 93, 191911 (2008). 